\title{
A Novel Encoding Algorithm for Textual Data Compression
}

\author{
Anas Al-okaily ${ }^{1, *}$ and Abdelghani Tbakhi ${ }^{1}$ \\ ${ }^{1}$ Department of Cell Therapy and Applied Genomics, King Hussein Cancer Center, Amman, 11941, Jordan \\ *anas.al-okaily@uconn.edu
}

\section{ABSTRACT}

Data compression is a fundamental problem in the fields of computer science, information theory, and coding theory. The need for compressing data is to reduce the size of the data so that the storage and the transmission of them become more efficient. Motivated from resolving the compression of DNA data, we introduce a novel encoding algorithm that works for any textual data including DNA data. Moreover, the design of this algorithm paves a novel approach so that researchers can build up on and resolve better the compression problem of DNA or textual data.

\section{Introduction}

The aim of compression process is to reduce the size of data as much as possible to save space and speed up transmission of data. There are two forms of compression processes: lossless and lossy. Lossless algorithms guarantee exact restoration of the original data, while lossy do not (due, for instance, to exclude unnecessary data such as data in video and audio where their loss will not be detected by users). The focus in this paper is the lossless compression.

Data can be in different formats such as text, numeric, images, audios, and videos. For textual data, which is the main focus in this paper, several encoding algorithms have been developed, namely and mainly, Huffman ${ }^{1}$ (with several versions, namely, minimum variance Huffman, canonical Huffman, length-limited Huffman, non-binary Huffman, and adaptive Huffman), Faller-Gallager-Knuth ${ }^{2}$ (an adaptive Huffman), Vitter ${ }^{3}$ (an adaptive Huffman), Shannon ${ }^{4}$, Shannon-Fano ${ }^{5}$, Shannon-FanoElias $^{6}$, Lempel-Ziv $^{7}$ (abbreviated as LZ with several extensions such as Lempel-Ziv-Welch ${ }^{8}$ (LZW), Lempel-Ziv-Stac ${ }^{9}$ (LZS), Lempel-Ziv-Oberhumer $^{10}$ (LZO), Lempel-Ziv-Storer-Szymanski ${ }^{11}$ (LZSS), and Lempel-Ziv-Ross-Williams ${ }^{12}$ (LZRW)), Burrows-Wheeler ${ }^{13}(\mathrm{BW})$, and Tunstall ${ }^{14}$ algorithms. There are several other algorithms that are named based on the type of process involved in the algorithm such as arithmetic encoding ${ }^{15}$, range encoding ${ }^{16}$, Move-to-Front encoding, ${ }^{17,18}$ (also referred as symbol ranking encoding), run-length-encoding ${ }^{19}$, delta encoding, unary encoding, context tree weighting encoding ${ }^{20}$, prediction by partial matching ${ }^{21}$, context mixing ${ }^{22}$, asymmetric numeral systems ${ }^{23}$ (referred also as asymmetric binary coding, finite state entropy is a variant of this entropy), LIPT $^{24}$, and dynamic Markov encoding 25 .

The main and common tools that implement one or more encoding algorithms and compress textual data are listed in Table 1. These tools are the ones that will be used to compare the results of the proposed algorithm with.

Compression algorithms can be classified based on the method type used in the algorithm. There are several method types used which are mainly: entropy, dictionary-based, predictive, and transform methods. A brief explanation of these methods is provided in the next two paragraphs but several and recent reviews and surveys that describe these methods are provided in several sources ${ }^{26-30}$.

The aim of entropy encoding is to represent the most frequent characters or words with less bits and vice versa, instead of using the same number of bits to represent every character as is used in standard representations such as ASCII ${ }^{31}$ encoding ( 8 bits for each character) and unicode encodings. Bits stands for binary digit and it is the basic unit of information in computing and digital communications with the value of 0 or 1 . Examples of entropy encoding include Huffman ${ }^{1}$ encoding and its family, Shannon $^{4}$, Shannon-Fano ${ }^{5}$, Shannon-Fano-Elias ${ }^{6}$, Tunstall $^{14}$, asymmetric numeral systems ${ }^{23}$, range encoding ${ }^{16}$, arithmetic encoding $^{15}$, and unary encoding. Predictive methods are based on employing the current decoded/decompressed data to predict the upcoming data. Context tree weighting encoding ${ }^{20}$, prediction by partial matching ${ }^{21}$, context mixing ${ }^{22}$, and dynamic Markov encoding ${ }^{25}$ are examples of algorithms that are based on prediction method.

The approach of dictionary encoding is to find the most repetitive patterns (characters or words) in the text and storing them in a dictionary then encode these patterns by its index in the dictionary. The dictionary can be built statically or dynamically. In static mode, same dictionary is used for all the compression process and is built ahead; this is suitable when a considerable prior knowledge about the text is available in advance. In the dynamic mode, the dictionary is built and updated during the compression process. This is achieved by employing a sliding window and then the dictionary is built from the previously 
encoded sequence within the sliding window. All the family of $\mathrm{LZ}^{7}$ algorithm and byte-pair algorithm ${ }^{32}$ apply this method. In transform methods, the text is converted to a representation that can be compressed more efficiently than the original representation. Examples mainly include move-to-front encoding, RLE encoding, delta encoding, LIPT, and BWT.

Textual data include genomics (DNA) data where there are several characteristics of this type of data. Firstly, the alphabet of DNA data consists of A, C, G, and T characters representing four nucleotides which are adenine, cytosine, guanine, and thymine; respectively. Secondly, DNA data contain repeats and palindromes. Thirdly, the size is very large. The human genome for instance consists of more than three billions of nucleotides. Moreover, sequencing genomic/DNA data is currently being performed in a daily basis around the globe and usually with high depth (30-100x). This generates in a daily basis a huge volume of data. Hence, many algorithms have been developed to handle the compression of this type of data. Compression algorithms involve two modes as introduced in ${ }^{66}$, algorithms that utilize reference genome/source (vertical mode) and algorithms that are reference-free (horizontal mode). The focus in this paper is on reference-free mode. DNA data represented as genomes are mainly stored in FASTA format, many algorithms have been developed for this format, namely, BIND $^{67}$, Biocompress ${ }^{68}$, Biocompress $2^{66}$, CBSTD $^{69}$, Cfact $^{70}$, CoGI $^{71}$, DELIMINATE $^{72}$, DNABIT $^{73}$, DNACompact $^{74}$, DNACOMPACT $^{75}$, DNACompress ${ }^{76}$, DNAC-SEB $^{77}$, DNAEcompress $^{78}$, DNAEnc $^{79}$, DNAPack $^{80}$, DNASC $^{81}$, DNAX $^{82}$, GeCo $^{83}$, $\mathrm{GeCo}^{84}{ }^{84}$, GENBIT ${ }^{85}$, GenCompress ${ }^{86}$, GeNML ${ }^{87}$, HUFFBIT $^{88}$, improved RLE ${ }^{89}$, JARVIS ${ }^{90}$, MFCompress ${ }^{91}$, modified

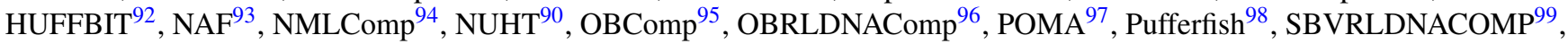
SeqCompress ${ }^{100}$, UHT ${ }^{101}, \mathrm{XM}^{102}$, modified RLE ${ }^{103}$, and other algorithms were proposed also in ${ }^{104-109}$. Several surveys and comparisons studies for DNA data are provided in the following sources ${ }^{77,91,110-112}$.

DNA data involve mainly genomics data where the genome (set of tens of chromosomes) is stored mainly in FASTA format ${ }^{113}$ (which is the main focus in this paper) and sequencing reads (millions/billions of reads where length of reads is in hundreds) which are stored with the quality of each base in mainly FASTQ format ${ }^{114}$. The number of bases that were sequenced since 1982 until June 2020 is 42,782,325,8901115. Many tools and algorithms have been developed to compress FASTQ

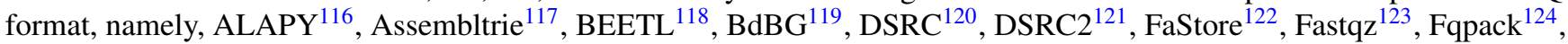

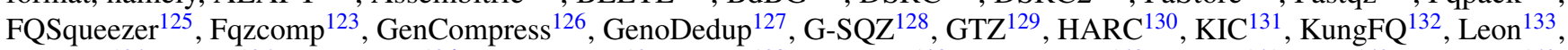

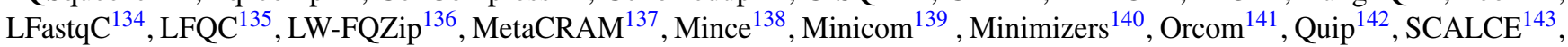
SeqDB $^{144}$, SlimGene ${ }^{145}$, SOLiDzipper ${ }^{146}$, SPRING $^{147}$, and in these papers ${ }^{148}$. Survey of this type of compression are published at $^{91,123}$.

Another common format used for storing/processing DNA data is SAM format ${ }^{149}$. Several algorithms have been developed to compress this format, namely, CRAM ${ }^{150}$, DeeZ ${ }^{151}$, Goby ${ }^{152}, \mathrm{HUGO}^{153}, \mathrm{NGC}^{154}$, Quip ${ }^{142}$, SAMcomp ${ }^{155}$, SAMtools ${ }^{149}$, SAMZIP ${ }^{156}$, and Scramble ${ }^{157}$. Surveys of this type of compression are published at ${ }^{91,123}$.

DNA data mostly contain characters with discrete uniform distribution (frequencies), hence, the compression process might not lead to high compression results as each character (base) will eventually assigned with almost same number of bits. If each character is represented by the same number of bits, then a naive and standard compression will be obtained which is not the aim of compression algorithms and not exploiting the repetitions contained in DNA data. As an example, instead of storing a sequence of twenty As followed by ten Ts, four Gs, then two As with 288 bits (36 bases $\times 8$ bits) using ASCII encoding; instead and using Huffman encoding, the character A will be represented by one bit (0), T with 2 bits (10), G with three bits (110), and $\mathrm{C}$ with three bits (111). The cost then for storing the sequence will be 58 bits instead of 288 bits. Now, if the characters in the sequence are distributed as 12 for A, 9 for T, 8 for G, and 6 for C, then Huffman encoding will assign 2 bits for each character (A:00. C: 01, G:10, and T:11, which lead to store the sequence with 72 bits); this is reasonable encoding but yet is not exploiting efficiently or optimally the repetitions in DNA sequence.

Now, given the small alphabet size of DNA data, which is better for the compression process, the main challenge however is the uniform distribution of the frequencies of the four characters. Motivated from resolving the compression problem for DNA data and from this aforementioned challenge, we introduce a novel encoding algorithm that is lossless, reference-free, resolves this challenge, and is applicable not only to DNA data to any textual data. By implementation, the algorithm shows better compression results indeed. As it has been a tradition to name the encoding algorithms by names, and to exploit this fact to honoring two great professors, Last author and Pramod Srivastava (Professor at Department of Immunology, University of Connecticut School of Medicine) who highly and exceptionally advised the first author in his academic career, kindly refer to this algorithm by Okaily-Srivastava-Tbakhi (OST).

\section{Methods}

One of the challenges in compressing DNA data is the uniform distribution of characters (bases). However, two observations can be drawn from a careful look to the DNA data. Firstly, looking to the regional (local) content, it's clear that many sub-sequences (let's say sub-sequences of length 100 bases) contain non-uniform/skewed distributions. Secondly, sequences which are similar and better if they are compressed and encoded together are distant from each other with distance longer than the length of the sliding window (length usually is of kilobytes) that is used in algorithms such as LZW, context weighting tree, predictive partial 
Table 1. Common general-purpose compression tools

\begin{tabular}{|c|c|c|c|}
\hline Tool & Algorithms used & Version & Last update \\
\hline $\mathrm{bcm}^{33}$ & based on BW algorithm & $\mathrm{v} 1.51$ & 2020 \\
\hline blzpack $^{34}$ & $\begin{array}{l}\text { based on brieflz library }{ }^{35} \text { where this library is a small and fast } \\
\text { open source implementation of a LZ style compression algo- } \\
\text { rithm }\end{array}$ & v1.3.0 & 2020 \\
\hline brotli $^{36}$ & $\begin{array}{l}\text { Google tool which combines LZ, Huffman encoding, and 2nd } \\
\text { order context modelling }\end{array}$ & v1.0.7 & 2018 \\
\hline $\mathrm{bsc}^{37}$ & based on block sorting & v3.1.0 & 2016 \\
\hline bzip2 $2^{38}$ & uses the BW algorithm & $\mathrm{v} 1.0 .8$ & 2019 \\
\hline cmix $^{39}$ & $\begin{array}{l}\text { a program aimed to optimizing compression ratio at the cost of } \\
\text { high CPU/memory usage }\end{array}$ & v18 & 2019 \\
\hline compress $^{40}$ & fast and simple compressor based on LZW algorithm & $\mathrm{v} 4.2 .4$ & 2011 \\
\hline Freeze $^{41}$ & combines LZSS and Huffman encoding & $\mathrm{v} 2.5 .0$ & 1993 \\
\hline gzip $^{42}$ & uses a combination of LZSS and Huffman coding & $\mathrm{v} 1.10$ & 2018 \\
\hline hook $^{43}$ & an implementation on dynamic Markov encoding & $\mathrm{v} 0.8 \mathrm{e}$ & 2007 \\
\hline $\begin{array}{l}\text { Huffman- } \\
\text { codec }^{44}\end{array}$ & based on Huffman encoding & $\mathrm{v} 1.0$ & 2020 \\
\hline lizard $^{45}$ & contains four methods such as fastLZ4 + Huffman & $\mathrm{v} 1.0$ & 2019 \\
\hline $\operatorname{lrzip}^{46}$ & $\begin{array}{l}\text { an extension of rzip but replaces bzip2 by LZMA, LZO, or no } \\
\text { second-stage }\end{array}$ & $\mathrm{v} 0.616$ & 2013 \\
\hline $1 z 4^{47}$ & $\begin{array}{l}\text { belongs to the LZ77 family of byte-oriented compression } \\
\text { schemes }\end{array}$ & v1.9.2 & 2019 \\
\hline $\mathrm{lzb}^{48}$ & based on hexadecimal and base 64 encoding/decoding & $\mathrm{v} 1.0$ & 2019 \\
\hline lzfse $^{49}$ & Apple tool that combines LZ and Finite State Entropy & $\mathrm{v} 1.0$ & 2017 \\
\hline lzip $^{50}$ & employs the Lempel-Ziv-Markov chain algorithm & $\mathrm{v} 1.19$ & 2017 \\
\hline lzop $^{51}$ & implements LZO algorithm & $\mathrm{v} 1.04$ & 2017 \\
\hline lzturbo $^{52}$ & compressor based on $1 \mathrm{Z77}$ algorithm & $\mathrm{v} 1.2$ & 2014 \\
\hline Nakamichi ${ }^{53}$ & based on LZSS algorithm & $\mathrm{v} 1.0$ & 2020 \\
\hline $\operatorname{pigz}^{54}$ & a parallel implementation of gzip & $\mathrm{v} 2.4$ & 2017 \\
\hline $\mathrm{ppm}^{55}$ & an implementation of prediction by partial matching & $\mathrm{v} 1.0$ & 2020 \\
\hline qzip $^{56}$ & $\begin{array}{l}\text { a gzip-like program which uses quicklz }{ }^{56} \text { compression library } \\
\text { where quicklz library is based on LZRW algorithm }\end{array}$ & $\mathrm{v} 0.2$ & 2011 \\
\hline rans_static ${ }^{57}$ & based on arithmetic encoding & v1.0 & 2016 \\
\hline rzip $^{58}$ & encodes large chunks of duplicated data then use bzip2 & v1.0.6 & 2010 \\
\hline $\operatorname{snzip}^{59}$ & $\begin{array}{l}\text { uses Snappy }{ }^{60} \text { which is a Google library based on ideas from } \\
\text { LZ77 }\end{array}$ & v1.0.4 & 2016 \\
\hline srank $^{61}$ & an implementation of Move-to-Front encoding (symbol ranking) & $\mathrm{v} 1.0$ & 1997 \\
\hline $\mathrm{xz}^{62}$ & $\begin{array}{l}\text { a variant algorithm of LZ77 with huge dictionary sizes and } \\
\text { special support for repeatedly used match distances where the } \\
\text { output is then encoded with a range encoder }\end{array}$ & v5.2.2 & 2015 \\
\hline Zlib $^{63}$ & variation of LZ algorithm & $\mathrm{v} 1.2 .11$ & 2017 \\
\hline zpipe $^{64}$ & is a ZLib implementation of gzip and deflate support & $\mathrm{v} 1.0$ & 2017 \\
\hline zstd $^{65}$ & $\begin{array}{l}\text { Facebook tool that combines a LZ with a large search window } \\
\text { and a fast entropy coding using both Finite State Entropy and } \\
\text { Huffman encoding }\end{array}$ & v1.4.5 & 2020 \\
\hline
\end{tabular}


matching, or dynamic Markov compression (even if these sequences are within the sliding window they are mostly distant from each other to a degree that makes encoding them costly and not efficient). These two observations motivated the design of a novel encoding algorithm.

Generally speaking, the algorithm is to scan the DNA data with non-overlapping windows, label the sequence within each window, concatenate it with the sequences in a bin correspondent to that label, and then output the label into an stream. Now, encode the labels of the bins based on, for instance, the number of the sequences in the bins; compress the stream of labels using the label codes. Then, compress the sequences in each bin using suitable compression algorithm depending on the content of the sequences in that bin. Finally, compress all the compression results (bins and stream of labels) together. The decompression process will be by firstly decompressing each bin and the stream of labels. Then, read the labels sequentially from the stream of labels, at each reading and using a counter for each bin, get the next sequence (of length same as the length of the non-overlapping window used during the compression process) from the bin of that label then increment the counter at that bin.

Compression algorithm. The steps of the algorithm for compressing a string $S$ of length $s$ and contains character from a fixed alphabet of length $\Sigma$, window length of $w$, sequence $Q L$ initialized to empty. The algorithm is as follows.

1. Scan $S$ with non-overlapping windows of length $w$, where at each window:

(a) Create label for the characters in the window so that this label classifies the sequence and where there is a bin correspondent to each label. The label can be set dynamically or designed in advance.

(b) Concatenate the label string with $Q L$, that stores the queue/order of the labels of the sequences, given that the string of each label must be unique.

(c) Concatenate the sequence of the window with the sequences in the bin correspondent to that label.

The time cost for this step is $O\left(\frac{s M}{w}\right)$ where $M$ is the cost for classifying each sequence of length $w$. If $M$ is less than or equal to $w$, then the cost of this step is $O(s)$. Moreover, the value of $w$ can be fixed or variable (variable so that the window is extended until the label of the sequence in the window matches one of the label of the bins). If $w$ is variable, then it must be added to $Q L$ (after the string of the label for each window) and encoded using a universal code for integers such as Levenshtein coding ${ }^{158}$, Elias coding ${ }^{159}$ (delta, gamma, or omega), exponential-Golomb code ${ }^{160}$, Fibonacci code ${ }^{161}$, Stout code ${ }^{162}$, or using a suitable encoding algorithm such as unary, binary, or Huffman encoding.

2. Once scanning $S$ is finished, encode all collected labels using Huffman encoding for instance. The time cost of this step is $l \log l$ where $l$ is the number of labels that the user or the implementer is set.

3. Compress $Q L$ using the encoding schema from the previous step. The time cost of this step is $O\left(\frac{s}{w}\right)$.

4. Compress the sequence in each bin with a compression algorithm suitable to the content of the sequences in the bin.

5. Compress/archive all resultant compressed files (bins and $Q L$ ) together.

\section{Decompression algorithm.}

1. Extract all files of compressed bins and compressed $Q L$.

2. Decompress all files (bins and $Q L$ ).

3. Initialize a counter for each bin.

4. Read from $Q L$ the strings of the labels sequentially where at each read extract the sequence of length $w$ starting from position equal to counter, increment counter by the value of $w$, then output the extracted sequence to the output stream.

Clearly, the time and memory cost of the decompression process is linear.

Analysis of the algorithm. The main process in the algorithm is clearly data-binning process. Each sequence of length $w$ (or of variable length) in the $S$ must be labeled and binned into the bin correspondent to that label. Then, the sequences which are sharing the same label and are binned together to the same bin are encoded using the same encoding algorithm. In addition, the sequences in each bin can be encoded using different encoding algorithm than the sequences in other bins. The design of the algorithm calls for several research questions which are still needed to be investigated. Given a set of sequences (concatenated together or not) and sharing similar characteristics (such as same Huffman tree), what is the efficient or optimal 
encoding solution for them? What is the optimal classification method to label the bins? What is the optimal number of bins to use? Is better to adopt a window length that is fixed, variable, or dependent on the text/genome length?

Data binning process has been used in solving compression problem but for sequencing reads not for genome sequences or textual data. The following tools, Assembltrie ${ }^{117}$, BEETL $^{118}$, BdBG $^{119}$, FaStore $^{122}$, HARC $^{130}$, Mince $^{138}$, Orcom $^{141}$, and SCALCE $^{143}$, apply data binning process but there are several differences between these algorithms and OST algorithm. Firstly, the inputs are already sequencing reads with short length (few hundreds bases) unlike the genomes or textual data. Secondly, sequencing of such reads are usually conducted at high coverage, hence, there are many redundancy in them and this is one of the motivations to apply binning process to compress these reads. Thirdly, none of these tools encode the the label of bins, in order to preserve the order of the reads in the original inputs, where this is due to the observation that the order of the reads in the input does not hold meaningful information so it can be omitted. However, a main step in OST algorithm is to encode the labels of the bins and record in $Q L$ the order these labels to preserve the order of the sequences so that during decompression the original DNA data can be restored. Lastly, applying binning process tom compress textual data (nor DNA/genomics data) was not addressed carefully in the literature.

The design of OST algorithm contributes in organizing and sorting the compression process by a divide-and-conquer methodology. The creation of bins for similar text and compress them instead of being dependent on a single encoding schema for all data as in entropy methods, or dependent on the previous sequence and its randomity (or differences with the upcoming sequence) to predict the upcoming sequences as in the prediction methods or to use in building a dictionary as in dictionary methods, or to transform the text into another text. This is supported with two observations as will be discussed and shown in the results section. Firstly, the bins with same label were compressed with same efficiency across different genomes and regardless of the content-type of the genomes (as an example repetitive or not repetitive as some genomes are known to be repetitive and others are not). Secondly, different compression algorithms seems to be better to compress different bins. Hence, OST design leads to more control and organizing of the content of the data being compressed, and what is more suitable to compress each bin rather than having the encoding/compression process dependent on the content of the data and dependent only on a single or a main algorithm.

OST-DNA This is the first implantation of OST algorithm in which the data is DNA data. The labels of the bins is computed using the encoding schema of Huffman tree of the content of the sequence. As an example, if the encoding schema is G:0, A:10, T:110, and C:111, then the label is GATC_1233 (1 indicates that G is encoded using 1 bit, A using 2 bits, and so on). The maximum number of labels (bins) hence is 65 .

Note that as the windows are non-overlapping, then each nucleotide in $S$ will be read $O(1)$ time. The cost for building Huffman tree is $\mathrm{O}(\Sigma \log \Sigma)$, where the number of times that Huffman trees needs to be built is $O\left(\frac{s}{w}\right)$ times; hence, the cost of building all Huffman trees is $O\left(\frac{s \Sigma \log \Sigma}{w}\right)$. Now, due to the pigeonhole principal and to allow obtaining non-uniform distribution for the nucleotides (characters) in $\Sigma$ within the content of the windows, the value of $w$ needs to be larger than the value of $\Sigma \log \Sigma$ noting that $\Sigma$ is a constant. Therefore, the time cost for this step is $O(s)$.

As $w$ value is fixed in this version of OST-DNA and is equal to...., Huffman tree is built once for each window's sequence. For the case of variable $w$ value (hence the window will be extending until the the label of the window's sequence match one of the labels of the bins), then it will not be efficient to compute Huffman tree for the whole sequence at every extension; instead, dynamic Huffman tree can be applied.

Lastly, in order to archive all the compressed bins and $Q L$, we tested several archiver tools such as ..... and decided to use $7 \mathrm{z}$ tool as it produced the best compression results.

\section{Results}

OST-DNA is implemented using python language. The code is not highly optimized in terms of speed and memory usage in both compression and decompression, but is sufficient as the main goal of this version of implementation is to proof the concept of the OST algorithm and conduct a trial testing and investigation. A set of genomes as listed in Table 2 and 3 , which is the same dataset used in ${ }^{110}$, were used for testing and comparing the results of OST-DNA with the results of the tools in Table 1.

A main metric of the performance of compression process is compression ratio which is equal to the size of the compressed file divided by the size of uncompressed (original) file. Other metrics also are considered in this paper which are compression speed $(\mathrm{MB} / \mathrm{s}$ ) which is equal to the size of uncompressed file (in $\mathrm{MB}$ ) divided by the compression time, and decompression speed $(\mathrm{MB} / \mathrm{s}$ ) which is equal to the size of the uncompressed file (in MB) divided by decompression time.

Firstly, all newlines and headers were removed from the genomes to obtain one sequence for the whole genome. Then we run all tools in Table 1 for all tested genomes. The results are provided in Table 4. For trialing, we used seven tools: bcm, brotli, bsc, bzip2, lrzip, lzip, and xz to compress the bins generated by OST-DNA algorithm. Moreover, we run each of these seven versions of OST-DNA when the value of $w$ is $25,50,100,125,150,200,300,400,500,600,800,1000,2000,3000,4000$, 5000, and 10000 and with different set of labels (as will described later). The minimum value obtained by these runs for each version on each tested genome is provided in Table 4 . 
Table 2. Genome sequence datasets

\begin{tabular}{|c|c|c|c|}
\hline Category & Organism & Accession & Size \\
\hline Virus & Gordoniaphage GAL1 $^{163}$ & GCF_001884535.1 & $50.7 \mathrm{kB}$ \\
\hline Bacteria & WS1 bacterium JGI 0000059-K21 164 & GCA_000398605.1 & $522 \mathrm{kB}$ \\
\hline Protist & Astrammina rara ${ }^{164}$ & GCA_000211355.2 & $1.71 \mathrm{MB}$ \\
\hline Fungus & Nosema ceranae ${ }^{164}$ & GCA_000988165.1 & $5.81 \mathrm{MB}$ \\
\hline Protist & Cryptosporidium parvumIowa II ${ }^{164}$ & GCA_000165345.1 & $9.22 \mathrm{MB}$ \\
\hline Protist & Spironucleus salmonicida ${ }^{164}$ & GCA_000497125.1 & $13.1 \mathrm{MB}$ \\
\hline Protist & Tieghemostelium lacteum ${ }^{164}$ & GCA_001606155.1 & $23.7 \mathrm{MB}$ \\
\hline Fungus & Fusarium graminearumPH-1 ${ }^{163}$ & GCF_000240135.3 & $36.9 \mathrm{MB}$ \\
\hline Protist & Salpingoeca rosetta ${ }^{164}$ & GCA_000188695.1 & $56.2 \mathrm{MB}$ \\
\hline Algae & Chondrus crispus ${ }^{164}$ & GCA_000350225.2 & $106 \mathrm{MB}$ \\
\hline Algae & Kappaphycus alvarezii ${ }^{164}$ & GCA_002205965.2 & $341 \mathrm{MB}$ \\
\hline Animal & Strongylocentrotus purpuratus ${ }^{163}$ & GCF_000002235.4 & $1.01 \mathrm{~GB}$ \\
\hline Plant & Picea abies ${ }^{164}$ & GCA_900067695.1 & 13.4 GB \\
\hline
\end{tabular}

Table 3. Other DNA datasets

\begin{tabular}{|c|c|c|c|c|}
\hline Dataset & $\begin{array}{l}\text { No. of se- } \\
\text { quences }\end{array}$ & Size & Source & Date \\
\hline \multirow[t]{2}{*}{ Mitochondrion $^{163}$} & 9,402 & $245 \mathrm{MB}$ & $\begin{array}{l}\text { RefSeq ftp: ftp://ftp.ncbi.nlm.nih. } \\
\text { gov/refseq/release/mitochondrion/ } \\
\text { mitochondrion.1.1.genomic.fna.gz }\end{array}$ & $\begin{array}{l}15 \text { March } \\
2019\end{array}$ \\
\hline & & & $\begin{array}{l}\text { ftp://ftp.ncbi.nlm.nih.gov/refseq/ } \\
\text { release/mitochondrion/mitochondrion. } 2 \text {. } \\
\text { 1.genomic.fna.gz }\end{array}$ & \\
\hline $\begin{array}{l}\text { NCBI Virus Com- } \\
\text { plete Nucleotide } \\
\text { Human }{ }^{165}\end{array}$ & 36,745 & $482 \mathrm{MB}$ & $\begin{array}{l}\text { NCBI Virus: https://www.ncbi.nlm.nih.gov/ } \\
\text { labs/virus/vssi/ }\end{array}$ & $\begin{array}{l}11 \quad \text { May } \\
2020\end{array}$ \\
\hline Influenza $^{166}$ & 700,001 & $1.22 \mathrm{~GB}$ & $\begin{array}{l}\text { Influenza Virus Database: ftp://ftp.ncbi.nih. } \\
\text { gov/genomes/INFLUENZA/influenza.fna. } \\
\text { gz }\end{array}$ & $\begin{array}{ll}27 & \text { April } \\
2019 & \end{array}$ \\
\hline Helicobacter ${ }^{164}$ & 108,292 & $2.76 \mathrm{~GB}$ & $\begin{array}{l}\text { NCBI Assembly: https://www.ncbi.nlm.nih. } \\
\text { gov/assembly }\end{array}$ & $\begin{array}{ll}24 & \text { April } \\
2019 & \end{array}$ \\
\hline
\end{tabular}


Table 4. Compression ration in percentage for all tested genomes using all tested tools. The indexes in the header are generated for each tested genome and provided in Table 5.

\begin{tabular}{|c|c|c|c|c|c|c|c|c|c|c|c|c|c|c|c|c|c|}
\hline & 1 & 2 & 3 & 4 & 5 & 6 & $I$ & o & 9 & 10 & 11 & 12 & 13 & 14 & 15 & 16 & 17 \\
\hline bcm & 23.92 & 3.83 & 4.85 & 3.56 & 23.71 & 24.06 & 3.40 & 24.72 & 21.71 & 24.05 & 20.71 & & 6.54 & & 2.36 & 16.28 & 23.68 \\
\hline & & 0.00 & & & & & $7.87 \mid$ & & & 37.86 & & & 36.96 & & 34.91 & & 37.54 \\
\hline brotli & 25.00 & 27.06 & 6.98 & $23.40 \mid$ & 26.27 & 24.39 & $4.13 \mid$ & $7.65 \mid$ & 23.73 & 19.47 & 15.16 & & 2.44 & 21.77 & 0.97 & 5.12 & 20.99 \\
\hline bsc & 24.38 & 24.28 & 25.09 & 23.96 & 24.15 & 24.19 & 3.81 & 24.88 & 21.88 & 23.45 & 20.10 & 24.90 & 5.63 & 21.26 & 2.04 & 13.60 & 23.70 \\
\hline bzip & 26.70 & 26.66 & 27.25 & $26.71 \mid$ & 26.75 & 26.86 & 26.50 & 27.05 & 24.15 & 26.89 & 24.71 & 27.22 & 11.72 & 23.38 & 5.56 & 26.41 & 26.85 \\
\hline free & 29.29 & 28.48 & 28.91 & 28.29 & 28.24 & 28.32 & 27.76 & 28.99 & 25.94 & 28.42 & 28.13 & 28.81 & 25.31 & 24.42 & 22.24 & 27.94 & 28.04 \\
\hline gzip & 29.16 & 28.80 & 29.07 & 28.74 & 28.68 & 28.53 & 28.16 & 28.99 & 26.12 & $28.47 \mid$ & 27.86 & 28.82 & 18.81 & $24.51 \mid$ & 11.50 & 28.33 & 28.38 \\
\hline lizar & 41.35 & 38.34 & 38.75 & 37.96 & 38.02 & 37.76 & $37.35 \mid$ & 38.87 & 34.46 & $37.71 \mid$ & 34.33 & 38.29 & 18.42 & 32.10 & 6.47 & 37.16 & 37.60 \\
\hline Irzip & 27.33 & 26.01 & 26.24 & 24.40 & 25.33 & 23.66 & $24.54 \mid$ & 26.39 & 22.65 & 22.10 & 16.75 & 25.27 & 3.58 & 21.17 & 1.41 & 6.72 & 20.26 \\
\hline lz4 & 67.79 & 55.73 & 55.84 & 55.56 & 55.55 & 55.15 & 55.32 & 56.06 & 50.09 & 55.12 & 54.76 & 55.78 & 38.49 & 47.25 & 29.46 & 55.08 & 55.15 \\
\hline lzb & 53.39 & 53.39 & 54.59 & 52.94 & 52.93 & 53.50 & 52.58 & 54.84 & 48.63 & $54.10 \mid$ & & & & & & & \\
\hline lzf: & 50.05 & 30.46 & 30.82 & 30.35 & 30.26 & & $0.04 \mid$ & 30.66 & & 3043 & 30.01 & & 27.55 & & 4.00 & 29.15 & 30.02 \\
\hline lzip & 27.27 & 26.24 & 26.60 & $24.41 \mid$ & 25.40 & 23.71 & 24.42 & 26.77 & 22.61 & 20.64 & 15.74 & 24.82 & 2.99 & 21.20 & 1.29 & 2 & 21.01 \\
\hline |zo| & 48.81 & 48.66 & 1020 & 48.61 & 48.49 & 48.76 & & & 44.22 & 48.92 & 48.47 & & $45.02 \mid$ & 42.52 & 40. & & 18 \\
\hline & 31.86 & 31.15 & 31.27 & 30.75 & 30.80 & 30.50 & 30.43 & 31.43 & 28.07 & 30.71 & 29.73 & & 16.19 & $26.21 \mid$ & 8.15 & & 30.39 \\
\hline nak & 40.17 & 34.93 & 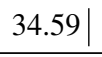 & 31.30 & 31.78 & 3056 & 3027 & 32.62 & & & & & & & & & \\
\hline OST & 24.12 & 23.94 & 24.05 & 23.59 & 23.71 & 25.95 & 23.40 & 24.72 & 21.71 & 23.76 & 19.78 & 24.69 & 6.02 & 21.22 & 2.35 & 14.63 & 16.64 \\
\hline OST & 25.23 & 24.73 & 24.92 & 23.34 & 24.06 & 22.69 & 23.52 & 24.81 & 21.72 & 17.97 & 14.11 & 22.95 & 2.17 & $20.17 \mid$ & 0.97 & 5.03 & 20.27 \\
\hline OST_bsc & 24.58 & 24.44 & 25.11 & 24.04 & 24.16 & 24.15 & 23.84 & 24.88 & 21.91 & 23.33 & $19.27 \mid$ & 24.80 & 5.17 & 21.27 & 2.03 & 12.59 & 23.28 \\
\hline OST_bzip2 & 26.90 & 26.72 & 27.28 & $26.67 \mid$ & 26.74 & 26.77 & 20.40 & 26.97 & 24.05 & 26.59 & 24.26 & 27.14 & 10.63 & $23.37 \mid$ & 5.47 & 19.36 & 21.22 \\
\hline OST_lrzip & 27.53 & 26.52 & 26.25 & 24.96 & 25.52 & 23.82 & 24.64 & 26.34 & 22.70 & $21.67 \mid$ & 16.28 & 25.06 & 3.29 & 21.22 & 1.41 & 6.90 & 17.60 \\
\hline & 27.48 & $26.67 \mid$ & 26.55 & $24.99 \mid$ & 25.57 & 23.86 & 24.01 & 26.12 & 22.60 & 20.38 & 15.28 & 24.59 & 2.73 & 2.96 & 1.29 & 6.15 & 20.67 \\
\hline & 27.46 & 26.50 & 26.32 & $24.97 \mid$ & 25.52 & 23.84 & $2+.00$ & 26.10 & 22.59 & 20.35 & 15.15 & 24.58 & 2.66 & 21.15 & 1.17 & 6.06 & 20.69 \\
\hline pbzip2 & 26.70 & 26.66 & 27.24 & 26.71 & 26.78 & 26.89 & 20.50 & 27.06 & 24.15 & 26.89 & 24.72 & 27.24 & 11.90 & 23.38 & 5.78 & 26.42 & 20.00 \\
\hline pigz & 29.16 & 28.84 & 29.11 & 28.77 & 28.71 & 28.56 & 28.20 & & & $28.51 \mid$ & 27.87 & 28.85 & 18.81 & 24.52 & 11.49 & 28.36 & 20.72 \\
\hline qzip & 42.75 & 42.98 & 43.54 & 43.20 & 42.98 & 43.23 & 42.43 & 43.84 & 39.08 & 43.44 & 42.64 & $44.07 \mid$ & 42.10 & 37.60 & 37.76 & 42.35 & 42.47 \\
\hline rzip & 26.87 & 26.68 & 27.27 & 26.74 & 26.80 & & 26.51 & 27.07 & & 26.90 & 24.78 & 27.23 & 12.17 & 23.38 & 6.05 & 26.41 & 26.86 \\
\hline snzip & 46.57 & 46.59 & $46.61 \mid$ & 46.64 & 46.48 & & 46.31 & & & 46.33 & 46.21 & 46.87 & 44.21 & 40.90 & 40.55 & 46.20 & 46.09 \\
\hline $\mathbf{x z}$ & 27.26 & 25.96 & 26.26 & 24.39 & & & 24.39 & & & 20.61 & 15.65 & & 2.92 & 21.19 & 1.16 & 6.27 & 21.05 \\
\hline zip & 29.84 & 28.87 & 29.10 & 28.75 & 28.68 & 28.53 & 28.16 & 28.99 & 26.12 & $28.47 \mid$ & 27.86 & 28.82 & 18.81 & $24.51 \mid$ & 11.50 & 28.33 & 28.38 \\
\hline zlib & 29.00 & 28.80 & 29.08 & 28.75 & 28.70 & 28.54 & 28.18 & 29.01 & 26.13 & 28.49 & 27.86 & 28.84 & 18.80 & $24.51 \mid$ & 11.49 & 28.34 & 28.40 \\
\hline zpaq & 41.01 & 37.46 & 36.34 & 34.87 & 34.76 & 34.49 & 34.31 & 35.83 & 31.67 & 34.67 & 31.62 & 35.16 & 11.94 & $29.41 \mid$ & 5.85 & 32.12 & 34.31 \\
\hline zpipe & 29.00 & 28.80 & 29.08 & 28.75 & 28.70 & 28.54 & 28.18 & 29.01 & 26.13 & 28.49 & 27.86 & 28.84 & 18.80 & 24.51 & 11.49 & 28.34 & 28.40 \\
\hline zstd & 31.18 & 30.27 & 30.87 & | & 30.01 & 29.69 & $29.50 \mid$ & 30.82 & 27.43 & 29.75 & $26.17 \mid$ & 30.33 & 5.70 & 25.84 & 2.55 & 26.21 & 29.88 \\
\hline
\end{tabular}


Table 5. Indexes of the genomes that are used in Table 4 along with the their sizes

\begin{tabular}{|r|l|l|}
\hline Index & Genome/DNA & Size \\
\hline 1 & Gordonia-phage-GAL1-GCF_001884535.1-2016-11-15. & $50.7 \mathrm{kB}$ \\
\hline 2 & WS1-bacterium-JGI-0000059-K21-GCA_000398605.1-2013-05-16. & $522 \mathrm{kB}$ \\
\hline 3 & Astrammina-rara-GCA_000211355.2-2011-04-27. & $1.71 \mathrm{MB}$ \\
\hline 4 & Nosema-ceranae-GCA_000988165.1-2015-05-05. & $5.81 \mathrm{MB}$ \\
\hline 6 & Spironucleus-salmonicida-GCA_000497125.1-2013-11-19. & $9.22 \mathrm{MB}$ \\
\hline 7 & Tieghemostelium-lacteum-GCA_001606155.1-2016-04-04. & $23.7 \mathrm{MB}$ \\
\hline 8 & Fusarium-graminearum-PH-1-GCF_000240135.3-2008-11-21. & $36.9 \mathrm{MB}$ \\
\hline 9 & Salpingoeca-rosetta-GCA_000188695.1-2011-02-10. & $56.2 \mathrm{MB}$ \\
\hline 10 & Chondrus-crispus-GCA_000350225.2-2013-05-22. & $106 \mathrm{MB}$ \\
\hline 11 & Mitochondrion-2019-03-15. & $245 \mathrm{MB}$ \\
\hline 12 & Kappaphycus-alvarezii-GCA_002205965.2-2018-03-09. & $341 \mathrm{MB}$ \\
\hline 13 & NCBI-Virus-Complete-Nucleotide-Human-2020-05-11. & $482 \mathrm{MB}$ \\
\hline 14 & Strongylocentrotus-purpuratus-GCF_000002235.4-2015-03-10. & $1.01 \mathrm{~GB}$ \\
\hline 15 & Influenza-2019-04-27. & $1.22 \mathrm{~GB}$ \\
\hline 16 & Helicobacter-2019-04-24. & $2.76 \mathrm{~GB}$ \\
\hline 17 & Picea-abies-GCA_900067695.1-2016-11-09. & $13.4 \mathrm{~GB}$ \\
\hline
\end{tabular}

\section{Data availability}

The source code of the tool is available at https://github.com/aalokaily/ost.

\section{Acknowledgements (not compulsory)}

\section{Additional information}

\section{References}

1. Huffman, D. A. et al. A method for the construction of minimum-redundancy codes. Proc. IRE 40, 1098-1101 (1952).

2. Knuth, D. E. Dynamic huffman coding. J. algorithms 6, 163-180 (1985).

3. Vitter, J. S. Design and analysis of dynamic huffman codes. J. ACM (JACM) 34, 825-845 (1987).

4. Shannon, C. E. A mathematical theory of communication. ACM SIGMOBILE mobile computing communications review 5, 3-55 (2001).

5. Fano, R. M. The transmission of information (Massachusetts Institute of Technology, Research Laboratory of Electronics ..., 1949).

6. Cover, T. M. Elements of information theory (John Wiley \& Sons, 1999).

7. Ziv, J. \& Lempel, A. A universal algorithm for sequential data compression. IEEE Transactions on information theory $\mathbf{2 3 ,}$ 337-343 (1977).

8. Welch, T. A. A technique for high-performance data compression. Computer 8-19 (1984).

9. Friend, R. C. Transport Layer Security (TLS) Protocol Compression Using Lempel-Ziv-Stac (LZS). RFC 3943, DOI: 10.17487/RFC3943 (2004).

10. Oberhumer, M. Lzo-a real-time data compression library. http://www. oberhumer. com/opensource/lzo/ (2008).

11. Storer, J. A. \& Szymanski, T. G. Data compression via textual substitution. J. ACM (JACM) 29, 928-951 (1982). 
12. Williams, R. N. An extremely fast ziv-lempel data compression algorithm. In [1991] Proceedings. Data Compression Conference, 362-371 (IEEE, 1991).

13. Burrows, M. \& Wheeler, D. J. A block-sorting lossless data compression algorithm. Citeseer (1994).

14. Tunstall, B. P. Synthesis of noiseless compression codes. Ph.D. thesis, Georgia Institute of Technology (1967).

15. Langdon, G. G. An introduction to arithmetic coding. IBM J. Res. Dev. 28, 135-149 (1984).

16. Martín, G. Range encoding: an algorithm for removing redundancy from a digitised message. In Video and Data Recording Conference, Southampton, 1979, 24-27 (1979).

17. Ryabko, B. Y. Data compression by means of a "book stack". Probl. Peredachi Informatsii 16, 16-21 (1980).

18. Bentley, J. L., Sleator, D. D., Tarjan, R. E. \& Wei, V. K. A locally adaptive data compression scheme. Commun. ACM 29, 320-330 (1986).

19. Capon, J. A probabilistic model for run-length coding of pictures. IRE Transactions on Inf. Theory 5, 157-163 (1959).

20. Willems, F. M., Shtarkov, Y. M. \& Tjalkens, T. J. The context-tree weighting method: basic properties. IEEE transactions on information theory 41, 653-664 (1995).

21. Cleary, J. \& Witten, I. Data compression using adaptive coding and partial string matching. IEEE transactions on Commun. 32, 396-402 (1984).

22. Mahoney, M. V. Adaptive weighing of context models for lossless data compression. Tech. Rep., Florida Tech (2005).

23. Duda, J. Asymmetric numeral systems: entropy coding combining speed of huffman coding with compression rate of arithmetic coding. arXiv preprint arXiv:1311.2540 (2013).

24. Awan, F. S. \& Mukherjee, A. Lipt: A lossless text transform to improve compression. In Proceedings International Conference on Information Technology: Coding and Computing, 452-460 (IEEE, 2001).

25. Cormack, G. V. \& Horspool, R. N. S. Data compression using dynamic markov modelling. The Comput. J. 30, 541-550 (1987).

26. Gopinath, A. \& Ravisankar, M. Comparison of lossless data compression techniques. In 2020 International Conference on Inventive Computation Technologies (ICICT), 628-633 (IEEE, 2020).

27. Kavitha, P. A survey on lossless and lossy data compression methods. Int. J. Comput. Sci. \& Eng. Technol. (IJCSET) 7 (2016).

28. squash. https://quixdb.github.io/squash-benchmark/.

29. Uthayakumar, J., Vengattaraman, T. \& Dhavachelvan, P. A survey on data compression techniques: From the perspective of data quality, coding schemes, data type and applications. J. King Saud Univ. Inf. Sci. (2018).

30. Kodituwakku, S. \& Amarasinghe, U. Comparison of lossless data compression algorithms for text data. Indian journal computer science engineering 1, 416-425 (2010).

31. Ascii code, howpublished $=$ https://www.ascii-code.com/, note $=$ accessed: 2020-06-30 .

32. Gage, P. A new algorithm for data compression. C Users J. 12, 23-38 (1994).

33. bcm. https://github.com/encode $84 / \mathrm{bcm}$.

34. blzpack. https://github.com/jibsen/brieflz/tree/master/example.

35. brieflz. https://github.com/jibsen/brieflz.

36. brotli. https://github.com/google/brotli.

37. bsc. https://github.com/IlyaGrebnov/libbsc.

38. bzip2. http://www.bzip.org/.

39. cmix. https://github.com/byronknoll/cmix.

40. compress. https://github.com/vapier/ncompress.

41. freeze. https://centos.pkgs.org/7/epel-x86_64/freeze-2.5.0-16.el7.x86_64.rpm.html.

42. Deutsch, P. et al. Gzip file format specification version 4.3 (1996).

43. hook. http://cs.fit.edu/ mmahoney/compression/hook.zip.

44. Huffman. https://github.com/Bestoa/huffman-codec. 
45. lizard. https://github.com/inikep/lizard.

46. lrzip. https://github.com/ckolivas/lrzip.

47. lz4. https://github.com/lz4/lz4.

48. lzb. https://github.com/faragon/lzb.

49. lzfse. https://developer.apple.com/documentation/compression/algorithm/lzfse.

50. lzip. https://www.nongnu.org/lzip/.

51. lzop. https://www.lzop.org/.

52. Lzturbo. https://sites.google.com/site/powturbo/.

53. Nakamichi. http://www.sanmayce.com/Nakamichi/.

54. pigz. https://zlib.net/pigz/.

55. ppm. https://github.com/rene-puschinger/ppm.

56. quicklz. https://github.com/robottwo/quicklz.

57. rans. https://github.com/jkbonfield/rans_static.

58. rzip. https://rzip.samba.org/.

59. snzip. https://github.com/kubo/snzip.

60. snappy. https://github.com/google/snappy.

61. srank. https://www.cs.auckland.ac.nz/ peter-f/FTPfiles/srank.c.

62. lzma/xz. https://tukaani.org/xz/format.html.

63. zlib. https://github.com/madler/zlib.

64. zpipe. https://github.com/skyformat99/zpipe.

65. zstd. https://github.com/facebook/zstd.

66. Grumbach, S. \& Tahi, F. A new challenge for compression algorithms: genetic sequences. Inf. Process. \& Manag. 30, 875-886 (1994).

67. Bose, T., Mohammed, M. H., Dutta, A. \& Mande, S. S. Bind-an algorithm for loss-less compression of nucleotide sequence data. J. biosciences 37, 785-789 (2012).

68. Grumbach, S. \& Tahi, F. Compression of dna sequences. In [Proceedings] DCC93: Data Compression Conference, 340-350 (IEEE, 1993).

69. Majumder, A. B. \& Gupta, S. Cbstd: a cloud based symbol table driven dna compression algorithm. In Industry Interactive Innovations in Science, Engineering and Technology, 467-476 (Springer, 2018).

70. Rivals, E., Delahaye, J.-P., Dauchet, M. \& Delgrange, O. A guaranteed compression scheme for repetitive dna sequences. In Proceedings of Data Compression Conference-DCC'96, 453 (IEEE, 1996).

71. Xie, X., Zhou, S. \& Guan, J. Cogi: Towards compressing genomes as an image. IEEE/ACM transactions on computational biology bioinformatics 12, 1275-1285 (2015).

72. Mohammed, M. H., Dutta, A., Bose, T., Chadaram, S. \& Mande, S. S. Deliminate-a fast and efficient method for loss-less compression of genomic sequences: sequence analysis. Bioinformatics 28, 2527-2529 (2012).

73. Rajarajeswari, P. \& Apparao, A. Dnabit compress-genome compression algorithm. Bioinformation 5, 350 (2011).

74. Gupta, A. \& Agarwal, S. A novel approach for compressing dna sequences using semi-statistical compressor. Int. J. Comput. Appl. 33, 245-251 (2011).

75. Li, P. et al. Dna-compact: Dna com pression based on a p attern-a ware c ontextual modeling t echnique. PloS one 8, e80377 (2013).

76. Chen, X., Li, M., Ma, B. \& Tromp, J. Dnacompress: fast and effective dna sequence compression. Bioinformatics 18, 1696-1698 (2002).

77. Mansouri, D., Yuan, X. \& Saidani, A. A new lossless dna compression algorithm based on a single-block encoding scheme. Algorithms 13, 99 (2020). 
78. Tan, L., Sun, J. \& Xiong, W. A compression algorithm for dna sequence using extended operations. J. Comput. Inf. Syst. 8, 7685-7691 (2012).

79. Pinho, A. J., Ferreira, P. J., Neves, A. J. \& Bastos, C. A. On the representability of complete genomes by multiple competing finite-context (markov) models. PloS one 6, e21588 (2011).

80. Behzadi, B. \& Le Fessant, F. Dna compression challenge revisited: a dynamic programming approach. In Annual Symposium on Combinatorial Pattern Matching, 190-200 (Springer, 2005).

81. Mishra, K. N. \& Aaggarwal, A. An efficient horizontal and vertical method for online dna sequence compression. Int. J. Comput. Appl. 3, 39-46 (2010).

82. Manzini, G. \& Rastero, M. A simple and fast dna compressor. Software: Pract. Exp. 34, 1397-1411 (2004).

83. Pratas, D., Pinho, A. J. \& Ferreira, P. J. Efficient compression of genomic sequences. In 2016 Data Compression Conference (DCC), 231-240 (IEEE, 2016).

84. Pratas, D., Hosseini, M. \& Pinho, A. J. Geco2: an optimized tool for lossless compression and analysis of dna sequences. In International Conference on Practical Applications of Computational Biology \& Bioinformatics, 137-145 (Springer, 2019).

85. Rajeswari, P. R., Apparo, A. \& Kumar, V. Genbit compress tool (gbc): A java-based tool to compress dna sequences and compute compression ratio (bits/base) of genomes. arXiv preprint arXiv:1006.1193 (2010).

86. Chen, X., Kwong, S. \& Li, M. A compression algorithm for dna sequences and its applications in genome comparison. Genome informatics 10, 51-61 (1999).

87. Korodi, G. \& Tabus, I. An efficient normalized maximum likelihood algorithm for dna sequence compression. $A C M$ Transactions on Inf. Syst. (TOIS) 23, 3-34 (2005).

88. RAJESWARI, P. R. et al. Huffbit compress-algorithm to compress dna sequences using extended binary trees. J. Theor. Appl. Inf. Technol. 13 (2010).

89. Ouyang, J., Feng, P. \& Kang, J. Fast compression of huge dna sequence data. In 2012 th International Conference on BioMedical Engineering and Informatics, 885-888 (IEEE, 2012).

90. Alyami, S. \& Huang, C.-h. Nongreedy unbalanced huffman tree compressor for single and multifasta files. J. Comput. Biol. (2019).

91. Hosseini, M., Pratas, D. \& Pinho, A. J. A survey on data compression methods for biological sequences. Information 7, 56 (2016).

92. Habib, N., Ahmed, K., Jabin, I. \& Rahman, M. M. Modified huffbit compress algorithm-an application of r. J. integrative bioinformatics 15 (2018).

93. Kryukov, K., Ueda, M. T., Nakagawa, S. \& Imanishi, T. Nucleotide archival format (naf) enables efficient lossless reference-free compression of dna sequences. Bioinformatics 35, 3826-3828 (2019).

94. Tabus, I., Korodi, G. \& Rissanen, J. Dna sequence compression using the normalized maximum likelihood model for discrete regression. In Data Compression Conference, 2003. Proceedings. DCC 2003, 253-262 (IEEE, 2003).

95. Mansouri, D. \& Yuan, X. One-bit dna compression algorithm. In International Conference on Neural Information Processing, 378-386 (Springer, 2018).

96. Roy, S., Mondal, S., Khatua, S. \& Biswas, M. An efficient compression algorithm for forthcoming new species. Int. J. Hybrid Inf. Tech 8, 323-332 (2015).

97. Zhu, Z., Zhou, J., Ji, Z. \& Shi, Y.-H. Dna sequence compression using adaptive particle swarm optimization-based memetic algorithm. IEEE Transactions on Evol. Comput. 15, 643-658 (2011).

98. pufferfish. https://github.com/alexholehouse/pufferfish.

99. Roy, S., Bhagot, A., Sharma, K. A. \& Khatua, S. Sbvrldnacomp: An effective dna sequence compression algorithm. Int. J. Comput. Sci. Appl 5, 73-85 (2015).

100. Sardaraz, M., Tahir, M., Ikram, A. A. \& Bajwa, H. Seqcompress: An algorithm for biological sequence compression. Genomics 104, 225-228 (2014).

101. Al-Okaily, A., Almarri, B., Al Yami, S. \& Huang, C.-H. Toward a better compression for dna sequences using huffman encoding. J. Comput. Biol. 24, 280-288 (2017). 
102. Cao, M. D., Dix, T. I., Allison, L. \& Mears, C. A simple statistical algorithm for biological sequence compression. In 2007 Data Compression Conference (DCC'07), 43-52 (IEEE, 2007).

103. Goel, S. et al. A compression algorithm for dna that uses ascii values. In 2014 IEEE International Advance Computing Conference (IACC), 739-743 (IEEE, 2014).

104. Keerthy, A. \& Priya, S. M. Lempel-ziv-welch compression of dna sequence data with indexed multiple dictionaries. Int. J. Appl. Eng. Res 12, 5610-5615 (2017).

105. Matsumoto, T., Sadakane, K. \& Imai, H. Biological sequence compression algorithms. Genome informatics 11, 43-52 (2000).

106. Chen, M., Li, R. \& Yang, L. Optimized context weighting for the compression of the un-repetitive genome sequence fragment. Wirel. Pers. Commun. 103, 921-939 (2018).

107. Chen, W.-H., Lu, Y.-W., Lai, F., Chien, Y.-H. \& Hwu, W.-L. Integrating human genome database into electronic health record with sequence alignment and compression mechanism. J. medical systems 36, 2587-2597 (2012).

108. Eric, P. V., Gopalakrishnan, G. \& Karunakaran, M. An optimal seed based compression algorithm for dna sequences. Adv. bioinformatics 2016 (2016).

109. Rexline, S., Aju, R. \& Trujilla, L. Higher compression from burrows-wheeler transform for dna sequence. Int. J. Comput. Appl 173, 11-15 (2017).

110. Kryukov, K., Ueda, M. T., Nakagawa, S. \& Imanishi, T. Sequence compression benchmark (scb) database-a comprehensive evaluation of reference-free compressors for fasta-formatted sequences. GigaScience 9, giaa072 (2020).

111. Bakr, N. S., Sharawi, A. A. et al. Dna lossless compression algorithms. Am. J. Bioinforma. Res. 3, 72-81 (2013).

112. Jahaan, A., Ravi, T. \& Panneer Arokiaraj, S. A comparative study and survey on existing dna compression techniques. Int. J. Adv. Res. Comput. Sci. 8 (2017).

113. Lipman, D. J. \& Pearson, W. R. Rapid and sensitive protein similarity searches. Science 227, 1435-1441 (1985).

114. Cock, P. J., Fields, C. J., Goto, N., Heuer, M. L. \& Rice, P. M. The sanger fastq file format for sequences with quality scores, and the solexa/illumina fastq variants. Nucleic acids research 38, 1767-1771 (2010).

115. genbank. https://www.ncbi.nlm.nih.gov/genbank/statistics/.

116. alapy. http://alapy.com/services/alapy-compressor/.

117. Ginart, A. A. et al. Optimal compressed representation of high throughput sequence data via light assembly. Nat. communications 9, 1-9 (2018).

118. Cox, A. J., Bauer, M. J., Jakobi, T. \& Rosone, G. Large-scale compression of genomic sequence databases with the burrows-wheeler transform. Bioinformatics 28, 1415-1419 (2012).

119. Wang, R., Li, J., Bai, Y., Zang, T. \& Wang, Y. Bdbg: a bucket-based method for compressing genome sequencing data with dynamic de bruijn graphs. PeerJ 6, e5611 (2018).

120. Deorowicz, S. \& Grabowski, S. Compression of dna sequence reads in fastq format. Bioinformatics 27, 860-862 (2011).

121. Roguski, Ł. \& Deorowicz, S. Dsrc 2-industry-oriented compression of fastq files. Bioinformatics 30, 2213-2215 (2014).

122. Roguski, Ł., Ochoa, I., Hernaez, M. \& Deorowicz, S. Fastore: a space-saving solution for raw sequencing data. Bioinformatics 34, 2748-2756 (2018).

123. Bonfield, J. K. \& Mahoney, M. V. Compression of fastq and sam format sequencing data. PloS one 8, e59190 (2013).

124. fqpack. https://sourceforge.net/projects/fqpack/.

125. Deorowicz, S. Fqsqueezer: k-mer-based compression of sequencing data. Sci. reports 10, 1-9 (2020).

126. Daily, K., Rigor, P., Christley, S., Xie, X. \& Baldi, P. Data structures and compression algorithms for high-throughput sequencing technologies. BMC bioinformatics 11, 514 (2010).

127. Cogo, V. V., Paulo, J. \& Bessani, A. Genodedup: Similarity-based deduplication and delta-encoding for genome sequencing data. IEEE Transactions on Comput. (2020).

128. Tembe, W., Lowey, J. \& Suh, E. G-sqz: compact encoding of genomic sequence and quality data. Bioinformatics 26, 2192-2194 (2010).

129. Xing, Y. et al. Gtz: a fast compression and cloud transmission tool optimized for fastq files. BMC bioinformatics $\mathbf{1 8}$, 233-242 (2017). 
130. Chandak, S., Tatwawadi, K. \& Weissman, T. Compression of genomic sequencing reads via hash-based reordering: algorithm and analysis. Bioinformatics 34, 558-567 (2018).

131. Zhang, Y., Patel, K., Endrawis, T., Bowers, A. \& Sun, Y. A fastq compressor based on integer-mapped k-mer indexing for biologist. Gene 579, 75-81 (2016).

132. Grassi, E., Di Gregorio, F. \& Molineris, I. Kungfq: A simple and powerful approach to compress fastq files. IEEE/ACM transactions on computational biology bioinformatics 9, 1837-1842 (2012).

133. Benoit, G. et al. Reference-free compression of high throughput sequencing data with a probabilistic de bruijn graph. BMC bioinformatics 16, 288 (2015).

134. Al Yami, S. \& Huang, C.-H. Lfastqc: A lossless non-reference-based fastq compressor. PLoS One 14, e0224806 (2019).

135. Nicolae, M., Pathak, S. \& Rajasekaran, S. Lfqc: a lossless compression algorithm for fastq files. Bioinformatics 31, 3276-3281 (2015).

136. Zhang, Y. et al. Light-weight reference-based compression of fastq data. BMC bioinformatics 16, 188 (2015).

137. Kim, M. et al. Metacram: an integrated pipeline for metagenomic taxonomy identification and compression. $B M C$ bioinformatics 17, 94 (2016).

138. Patro, R. \& Kingsford, C. Data-dependent bucketing improves reference-free compression of sequencing reads. Bioinformatics 31, 2770-2777 (2015).

139. Liu, Y., Yu, Z., Dinger, M. E. \& Li, J. Index suffix-prefix overlaps by (w, k)-minimizer to generate long contigs for reads compression. Bioinformatics 35, 2066-2074 (2019).

140. Roberts, M., Hayes, W., Hunt, B. R., Mount, S. M. \& Yorke, J. A. Reducing storage requirements for biological sequence comparison. Bioinformatics 20, 3363-3369 (2004).

141. Grabowski, S., Deorowicz, S. \& Roguski, Ł. Disk-based compression of data from genome sequencing. Bioinformatics 31, 1389-1395 (2015).

142. Jones, D. C., Ruzzo, W. L., Peng, X. \& Katze, M. G. Compression of next-generation sequencing reads aided by highly efficient de novo assembly. Nucleic acids research 40, e171-e171 (2012).

143. Hach, F., Numanagić, I., Alkan, C. \& Sahinalp, S. C. Scalce: boosting sequence compression algorithms using locally consistent encoding. Bioinformatics 28, 3051-3057 (2012).

144. Howison, M. High-throughput compression of fastq data with seqdb. IEEE/ACM transactions on computational biology bioinformatics 10, 213-218 (2012).

145. Kozanitis, C., Saunders, C., Kruglyak, S., Bafna, V. \& Varghese, G. Compressing genomic sequence fragments using slimgene. J. Comput. Biol. 18, 401-413 (2011).

146. Jeon, Y. J., Park, S. H., Ahn, S. M. \& Hwang, H. J. Solidzipper: A high speed encoding method for the next-generation sequencing data. Evol. Bioinforma. 7, EBO-S6618 (2011).

147. Chandak, S., Tatwawadi, K., Ochoa, I., Hernaez, M. \& Weissman, T. Spring: a next-generation compressor for fastq data. Bioinformatics 35, 2674-2676 (2019).

148. Bhola, V., Bopardikar, A. S., Narayanan, R., Lee, K. \& Ahn, T. No-reference compression of genomic data stored in fastq format. In 2011 IEEE International Conference on Bioinformatics and Biomedicine, 147-150 (IEEE, 2011).

149. Li, H. et al. The sequence alignment/map format and samtools. Bioinformatics 25, 2078-2079 (2009).

150. Fritz, M. H.-Y., Leinonen, R., Cochrane, G. \& Birney, E. Efficient storage of high throughput dna sequencing data using reference-based compression. Genome research 21, 734-740 (2011).

151. Hach, F., Numanagic, I. \& Sahinalp, S. C. Deez: reference-based compression by local assembly. Nat. methods 11, 1082-1084 (2014).

152. Campagne, F., Dorff, K. C., Chambwe, N., Robinson, J. T. \& Mesirov, J. P. Compression of structured high-throughput sequencing data. PLoS one 8, e79871 (2013).

153. Li, P. et al. Hugo: Hierarchical multi-reference genome compression for aligned reads. J. Am. Med. Informatics Assoc. 21, 363-373 (2014).

154. Popitsch, N. \& von Haeseler, A. Ngc: lossless and lossy compression of aligned high-throughput sequencing data. Nucleic acids research 41, e27-e27 (2013). 
155. samcomp. https://sourceforge.net/projects/samcomp/.

156. Sakib, M. N., Tang, J., Zheng, W. J. \& Huang, C.-T. Improving transmission efficiency of large sequence alignment/map (sam) files. PloS one 6, e28251 (2011).

157. Bonfield, J. K. The scramble conversion tool. Bioinformatics 30, 2818 (2014).

158. Levenshtein. http://www.compression.ru/download/articles/int/levenstein_1968_on_the_redundancy_and_delay.pdf, author=Vladimir Levenshtein, year=1968.

159. Elias, P. Universal codeword sets and representations of the integers. IEEE transactions on information theory 21, 194-203 (1975).

160. Salomon, D. Data compression: the complete reference (Springer Science \& Business Media, 2004).

161. Fraenkel, A. S. \& Kleinb, S. T. Robust universal complete codes for transmission and compression. Discret. Appl. Math. 64, 31-55 (1996).

162. Stout, Q. Improved prefix encodings of the natural numbers (corresp.). IEEE Transactions on Inf. Theory 26, 607-609 (1980).

163. O'Leary, N. A. et al. Reference sequence (refseq) database at ncbi: current status, taxonomic expansion, and functional annotation. Nucleic acids research 44, D733-D745 (2016).

164. Clark, K., Karsch-Mizrachi, I., Lipman, D. J., Ostell, J. \& Sayers, E. W. Genbank. Nucleic acids research 44, D67-D72 (2016).

165. Brister, J. R., Ako-Adjei, D., Bao, Y. \& Blinkova, O. Ncbi viral genomes resource. Nucleic acids research 43, D571-D577 (2015).

166. Bao, Y. et al. The influenza virus resource at the national center for biotechnology information. J. virology 82, 596-601 (2008). 